\title{
Spectroscopy of Light Emission from a Scanning Tunneling Microscope in Air
}

\author{
R. Péchou (*), R. Coratger, C. Girardin, F. Ajustron and J. Beauvillain \\ Centre d'Élaboration des Matériaux et d'Études Structurales, 29 rue J. Marvig, \\ B.P. 4347, 31055 Toulouse Cedex, France
}

(Received 3 May 1996, revised 5 July 1996, accepted 2 September 1996)

\begin{abstract}
PACS.61.16.Ch - Scanning probe microscopy: scanning tunneling atomic force, scanning optical, magnetic force, etc.

PACS.78..66.-w - Optical properties of specific thin films, surfaces, and low-dimensional structures: superlattices quantum well structures, multilayers acid microparticles
\end{abstract}

\begin{abstract}
Light emission has been detected at the tip-sample junction of a Scanning Tunneling Microscope (S.T.M.) in air on noble metallic surfaces. A spectroscopic study of emitted photons for $\mathrm{Au}-\mathrm{Au}$ and PtIr-Au tunneling junctions is presented. The general aspect of the spectra depends on the materials used in the junctions; a study of the spectra as a function of tunneling current and surface bias voltage reveals similar and reproducible characteristics.
\end{abstract}

Résumé. - Une émission de lumière a été détectée au niveau de la jonction pointe-surface d'un microscope à effet tunnel dans l'air sur des surfaces de métaux nobles. Une étude spectroscopique des photons émis par des jonctions tunnel $\mathrm{Au}-\mathrm{Au}$ et PtIr-Au est présentée. L'aspect général des spectres dépend des matériaux utilisés ; une étude en fonction du courant tunnel et de la tension de polarisation de la jonction révèle des caractéristiques similaires et reproductibles.

\section{Introduction}

Since the pioneering work of Gimzewski et al. in 1988 [1], light emission stimulated by a Scanning Tunneling Microscope (S.T.M.) has proven to be a new way of investigating surface properties with a high lateral resolution. In addition, this technique allows excitations of solids to be studied at a subnanometric scale using a high-resolution imaging tool [2-6].

This phenomenon, previously by Lambe and McCarthy detected on polarized planar junctions [7], involves various theoretical interpretations depending on the nature of materials concerned. Light emission from noble metallic surfaces has been attributed to interface plasmon modes excited by inelastic tunneling electrons. These localized Tip Induced Plasmon (T.I.P.) modes are expected to radiate in photons if the translational invariance $k_{/ /}$of their momentum is broken by surface roughness or by the tip itself [8-10]. In the case of semiconductor samples, electron-hole recombinations are responsible for photon emission $[3,11]$.

$\left(^{*}\right)$ Author for correspondence

(C) Les Éditions de Physique 1996 
Using this light emission as an imaging technique, photonic mappings of scanned surfaces have been drawn up and a few interesting properties have been evidenced such as the control of light emission in air [12-14].

This paper presents a frequency spectroscopic study on Au-Au and PtIr-Au tunneling junctions in air. For a given Au surface and tip material, the wavelength components of the emitted light strongly depends on the tip used. However, reproducible phenomena can be observed when tunneling current or bias voltage are increased. The wavelength of the emitted photons is found to be independent of tunneling current but spectra depends on the materials of tunneling junctions and on applied bias voltage, i.e. energy of tunneling electrons. For each tunneling junction, the average spectrum at fixed bias voltage has been obtained from a set of recorded spectra.

\section{Experimental Set-Up}

The experimental set-up has already been described elsewhere [12-14]. A customized "pocketsize" STM operating in the constant current mode was adapted for photon detection. The emitted light was collected by five optic fibers with a core diameter of $1 \mathrm{~mm}$ each placed as close to the tip-sample junction as possible (about $1 \mathrm{~mm}$ ) and oriented $45^{\circ}$ relative to the normal of the sample surface. Two optic fibers were connected to photomultipliers and three to a spectrometer.

Photomultipliers (PM) (Hamamatsu R2949) operate in the pulse counting mode with a dark count rate of 1 count per second at $255 \mathrm{~K}$ in the $185-900 \mathrm{~nm}$ range, the complete range being used to draw up photon mappings of scanned areas.

The spectrometer consists of an Optic Multichannel Analyser (EG\&G, O.M.A.1245) connected with a C.C.D. detector cooled at $173 \mathrm{~K}$ and operating in the $300-1050 \mathrm{~nm}$ range. The scattering system is a grating blazed at $750 \mathrm{~nm}$. The effective spectral resolution of the spectrometer is of $2 \mathrm{~nm}$. Spectra are integrated over $30 \mathrm{~s}$ to increase signal-to-noise ratio. The response curve of our measurement system (optic fibers, scattering system and C.C.D. detector) has been taken into account and recorded spectra were corrected at each wavelength by an appropriate function calculated from the expected black body spectrum of a carbon filament lamp.

This experimental set-up allows simultaneous acquisition of S.T.M. topography, related photon mapping and emission spectrum of the scanned area.

Samples were monocrystalline gold films evaporated to a thickness of $50 \mathrm{~nm}$ on freshly cleaved mica sheets heated at $623 \mathrm{~K}$ during 24 hours at $10^{-7}$ Torr [15]. A low deposition rate of $0.1 \mathrm{~nm} \mathrm{~s}^{-1}$ allowed quasi-epitaxy of the gold layer along the [111] direction on the hot mica substrate as demonstrated by $\mathrm{X}$-ray diffraction experiments. These samples exhibited flat terraces typically $100 \times 100 \mathrm{~nm}^{2}$ in size separated by $2 \mathrm{~nm}$ high steps. Tips were prepared by cutting $0.25 \mathrm{~mm}$ diameter $\mathrm{Au}$ or PtIr wires.

\section{Experimental Results and Discussions}

2.1. Emission Spectra as a Function of Tunneling Current. -- Several spectra were recorded for $\mathrm{Au}-\mathrm{Au}$ tunneling junctions in air in the $5 \mathrm{nA}-100 \mathrm{nA}$ range, bias voltage being set to $1.8 \mathrm{~V}$. These spectra are shown in Figure 1. The same study has been done for PtIr$\mathrm{Au}$ tunneling junction and spectra were recorded in the $10 \mathrm{nA}-100 \mathrm{nA}$ range at a surface bias voltage of $1.8 \mathrm{~V}$, as shown in Figure 2. The overall emission rate of a PtIr-Au tunneling junction being weaker than that of an Au-Au junction, the spectra given in Figure 2 have been integrated over $60 \mathrm{~s}$. 


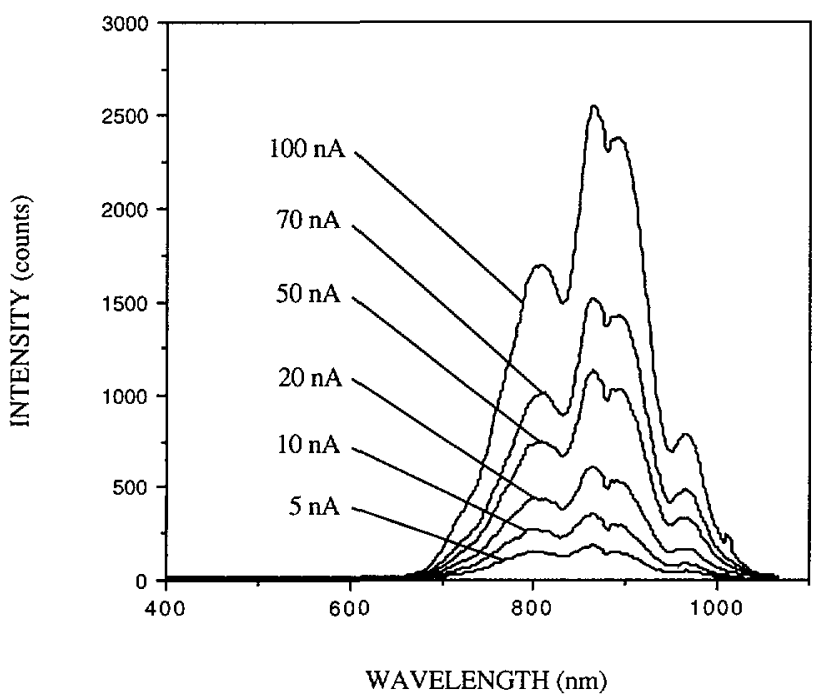

Fig. 1. - Emission spectra of an Au surface scanned by an Au-tip at various tunneling currents in the $5 \mathrm{nA}-100 \mathrm{nA}$ range. Bias voltage has been set to $1.8 \mathrm{~V}$. An increase in emission rate with tunneling current is observed.

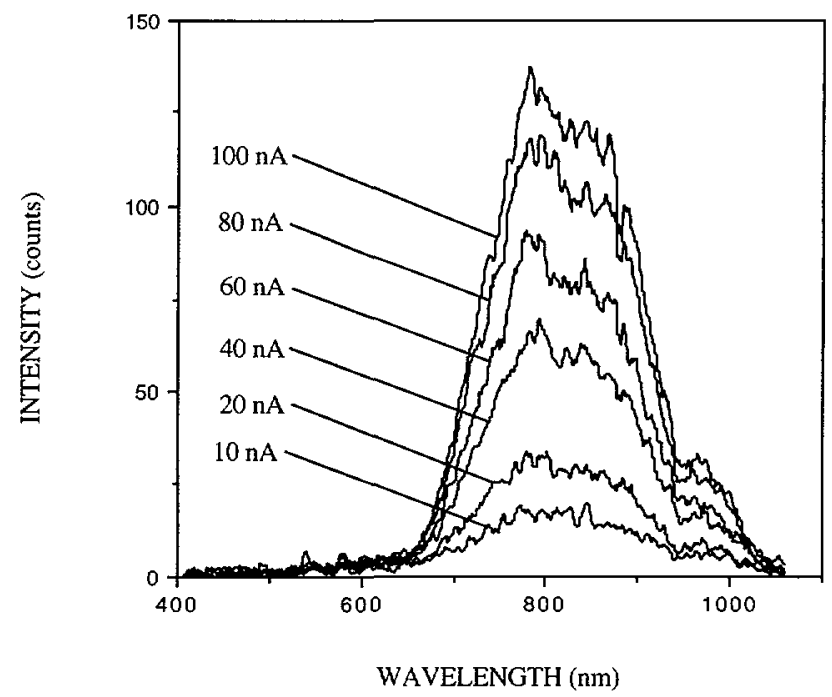

Fig. 2. - Emission spectra of an Au surface scanned by a PtIr-tip at various tunneling currents in the $10 \mathrm{nA}-100 \mathrm{nA}$ range. Bias voltage has been set to $1.8 \mathrm{~V}$.

An increase in emission rate is clearly evidenced but emission wavelengths remain unchanged when the tunneling current is increased. The amplitude of each spectrum component is simply increased as the tunneling current. Then, the number of emitted photons increases proportionately with the number of injected electrons, thereby evidencing that the electron is the particle responsible for light emission in an S.T.M. This experimental result had already be 


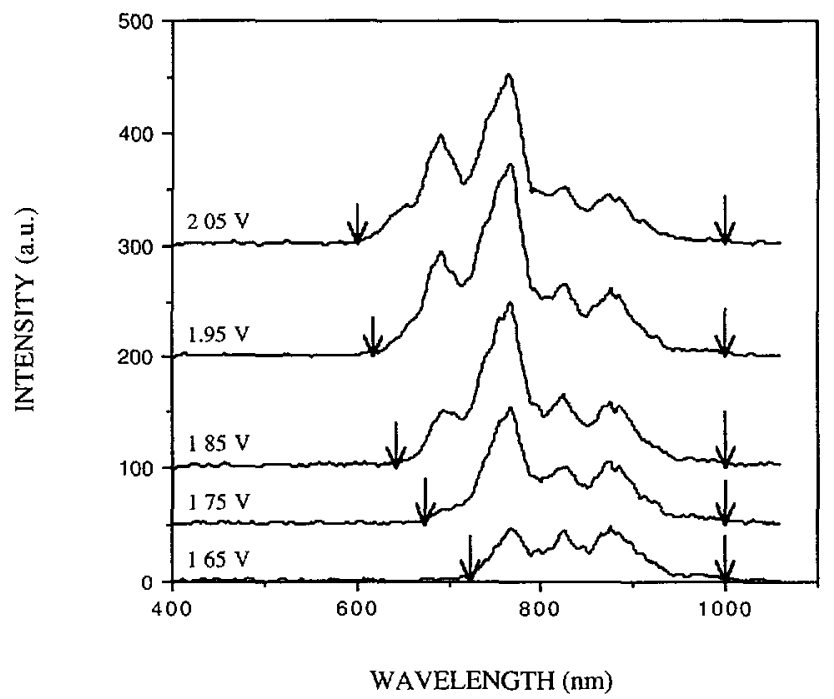

Fig. 3. - Emission spectra of an Au surface scanned by an Au-tip at various bias voltages in the $1.65 \mathrm{~V}-2.05 \mathrm{~V}$ range. Tunneling current has been set to $10 \mathrm{nA}$. Cut-offs of each spectrum are indicated by arrows.

mentioned by Sivel et al. [14]. The basic working principle of a S.T.M. relies on the strong dependence of tunneling current $I_{\mathrm{t}}$ on tip-sample distance $d_{\mathrm{tip}-\text { sample }}$. Thus, changes of $I_{\mathrm{t}}$ in the $5 \mathrm{nA}-100 \mathrm{nA}$ range (at a fixed bias voltage of $1.8 \mathrm{~V}$ ) are expected to cause important modifications of $d_{\mathrm{tip}-\text { sample: }}$ a variation of about $0.2 \mathrm{~nm}$ is expected in the one-dimension model of tunneling current [16]. Such a variation cannot be considered as a fluctuation around an

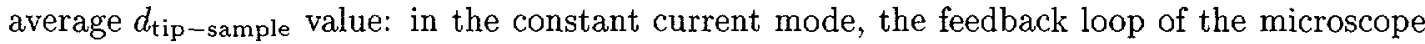
does not allow such variations in the tip-sample distance. Emission wavelengths are thus found

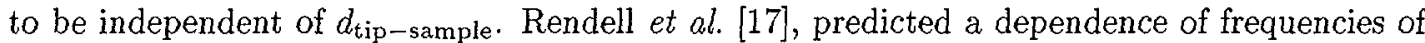
localized T.I.P. modes with $\left(d_{\text {tip-sample }}\right)^{1 / 4}$ The energy of the emitted photons being equal to the energy of the excited eletromagnetic modes [18], variations should be observed in the spectra positions. This model seems to be ill-suited when it comes to correctly describing the phenomenon.

\subsection{Emission Spectra as a Function of Bias Voltage}

2.2.1. Experimental Results. - Several spectra were recorded for Au-Au tunneling junctions in air in the $1.65 \mathrm{~V}-2.05 \mathrm{~V}$ range, tunneling current being set to $10 \mathrm{nA}$. These spectra are shown in Figure 3. The overall emission rate of the tunneling junction is found to increase with applied bias voltage in this voltage range. New high-energy components appear in the spectrum when bias voltage is increased: a $700 \mathrm{~nm}$-peak can easily be observed when bias voltage reaches $1.8 \mathrm{~V}$ and a shoulder centered at about $660 \mathrm{~nm}$ can be seen in the $2.05 \mathrm{~V}$ spectrum. An increase in the high energy component amplitude (the $770 \mathrm{~nm}$-peak and, when bias voltage reaches $1.85 \mathrm{~V}$, the $700 \mathrm{~nm}$-peak) with bias voltage is also clearly shown: the $770 \mathrm{~nm}$-peak becomes three times greater than the low-energy components when bias voltage increases from $1.65 \mathrm{~V}$ up to $2.05 \mathrm{~V}$. Cut-offs of each spectrum are indicated in Figure 3 by arrows. In this voltage range. a blue shift of high-energy cut-offs is also evidenced when increasing bias voltage. 


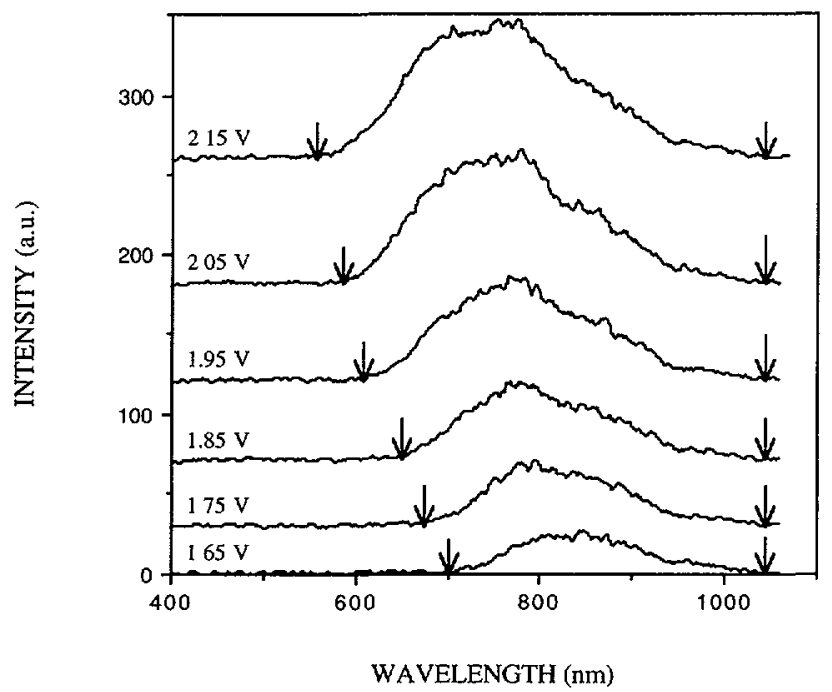

Fig. 4. - Emission spectra of an Au surface scanned by a PtIr-tip at various bias voltages in the $1.65 \mathrm{~V}-2.15 \mathrm{~V}$ range. Tunneling current has been set to $20 \mathrm{nA}$. Cut-offs of each spectrum are indicated by arrows.

In the case of PtIr-Au tunneling junctions, several spectra were recorded in air in the $1.65 \mathrm{~V}$ - $2.15 \mathrm{~V}$ range, tunneling current being set to $20 \mathrm{nA}$ owing to the weaker emission rate of PtIr-Au junctions. These spectra, shown in Figure 4, differ from those obtained for Au-Au tunneling junctions. The different components consist of bumps rather than sharp peaks, but the phenomena obtained in the case of $\mathrm{Au}-\mathrm{Au}$ tunneling junctions when increasing bias voltage remain the same for PtIr-Au junctions. For a sample bias voltage of $1.65 \mathrm{~V}$, the spectrum is centered at about $820 \mathrm{~nm}$ while it is located at about $750 \mathrm{~nm}$ when sample bias voltage reaches $2.15 \mathrm{~V}$.

2.2.2. Discussion. - As the wavelengths of the different components of recorded spectra remain unchanged when bias voltage is increased, emission frequencies are independent of bias voltage. The fundamental mechanism of light emission is thus found to be independent of bias voltage. However, new spectral components appear in the $1.65 \mathrm{~V}-2.05 \mathrm{~V}$ range. When bias voltage is increased, the potential barrier and thereby the Injected Electron Energy Distribution (I.E.E.D.) are modified. The presence (or absence) of given peaks is therefore closely linked to the I.E.E.D..

Experimental high-energy cut-offs are in good agreement with calculated ones ( $\lambda_{\text {cut-off }}=$

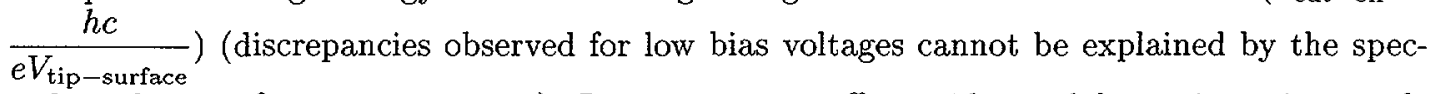
tral resolution of our spectrometer). Low-energy cut-offs are identical for a given tip-sample junction.

In the case of PtIr-Au tunneling junctions, the bumpiness observed on recorded spectra shows that at a given bias voltage the I.E.E.D. is different for an Au-tip and for a PtIr-tip. To account for this, two hypotheses can be put forward. The I.E.E.D. is expected to be different between an Au-tip and a PtIr one as energy levels are different in these two materials. Besides, injected electrons may lose part of their energy through a contaminant layer at the PtIr-tip apex and the low-energy tail of the I.E.E.D. can thereby be lengthened. A given tip-sample 


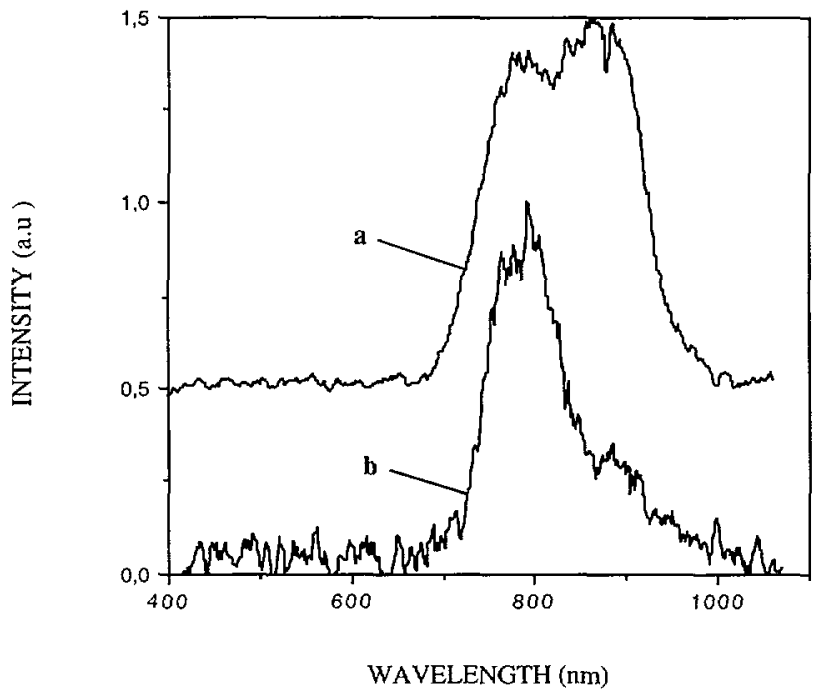

Fig. 5. - Normalized spectra of the light emitted by a $200 \times 200 \mathrm{~nm}^{2}$ Au area scanned with two different Au-tips at a surface bias voltage of $1.8 \mathrm{~V}$, a tunneling current of $15 \mathrm{nA}$ and a scan rate of $0.625 \mathrm{~Hz}$. Two distinct peaks are visible at about $790 \mathrm{~nm}$ and $880 \mathrm{~nm}$ on spectrum (a). while spectrum (b) exhibits a sharp peak at $790 \mathrm{~nm}$.

configuration therefore leads to a given I.E.E.D. and consequently a given spectrum.

These results have to be compared with spectra obtained by Berndt [18] in an U.H.V.S.T.M.. In these experiments, Au samples were scanned by W-tips. Recorded spectra evidenced a main spectral peak located in the $600-700 \mathrm{~nm}$ region. A blue shift of this spectral peak was also observed in the $2.0 \mathrm{~V}-4.0 \mathrm{~V}$ range. Differences observed in emission wavelengths may partially be due to the fact that our experiment is conducted in air: the presence of contaminants on the tip apex and on the sample surface may cause important modifications in the tunneling barrier particularly concerning the I.E.E.D. and Local Densities Of States (L.D.O.S.) of the sample surface.

\section{Average Spectra at a Given Bias Voltage}

Spectra shown in Figure 5 were recorded during scans of a $200 \times 200 \mathrm{~nm}^{2}$ Au surface with two different Au-tips at a surface bias voltage of $1.8 \mathrm{~V}$. The first spectrum (a) clearly evidences two discrete components centered at about $790 \mathrm{~nm}$ and $880 \mathrm{~nm}$ respectively while the second one (b) exhibits a sharp peak centered at $790 \mathrm{~nm}$. Figure 5 clearly shows that two spectra obtained from the same sample surface scanned with two different tips made with the same material are different. The same remarks can be made concerning spectra taken from Figures 1 and 3 (these spectra have been obtained from two different tunneling junctions made with the same materials and under very close tunneling conditions). To average this, a large set of experimental spectra is required to study light emission from a given type of tunneling junction. 45 spectra were recorded for different areas scanned with different Au-tips on different Aumonocrystalline samples at a fixed bias voltage of $1.8 \mathrm{~V}$ and a fixed tunneling current of $10 \mathrm{nA}$. An average spectrum has been obtained by summing up these 45 spectra. This normalized average spectrum is shown in Figure 6a. A given spectrum of emitted light corresponds to 


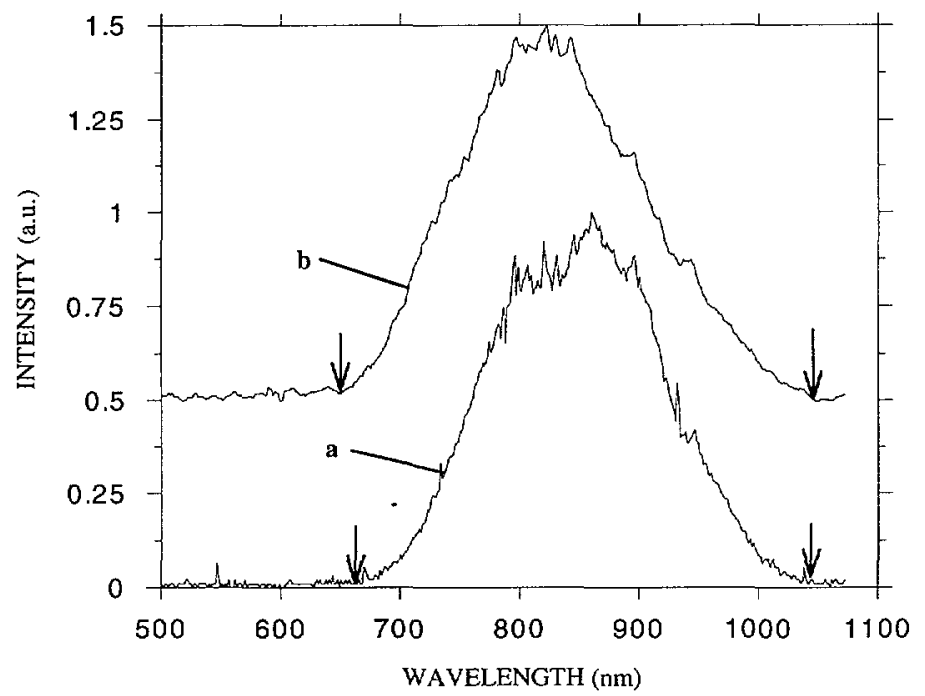

Fig. 6. - Normalized average spectra of light emitted by an Au-Au (a) and a PtIr-Au (b) tunneling junction at a fixed bias voltage of $1.8 \mathrm{~V}$, tunneling current being set at $10 \mathrm{nA}$. These spectra have been obtained from 45 spectra recorded during scans of different Au surfaces with different Au and PtIr-tips.

a given tunneling configuration with a given tip geometry, tip apex oxidization state and contamination state of the scanned area (i.e. characteristics of a given spectrum do not depend only on the sample surface). These tunneling conditions impose a given I.E.E.D. and L.D.O.S. and consequently a given spectrum shape (the fine structure observed on emission spectra could be linked to these peculiar I.E.E.D. and L.D.O.S.). By summing up all these specific spectra. these characteristic effects are toned over and the average spectrum can better be described as a large peak in the near-infrared region than as the sum of two or three distinct components.

As for Au-Au tunneling junctions, 45 spectra have been recorded for different PtIr-Au tunneling junctions at a fixed bias voltage of $1.8 \mathrm{~V}$ and a tunneling current of $10 \mathrm{nA}$. These recorded spectra have been summed up to obtain the normalized average spectrum of Figure $6 \mathrm{~b}$. Two differences can be observed between the spectra of Figure 6 . First of all, the low-energy sides of these average spectra are different. To account for this, the two hypotheses previously mentioned (modifications of I.E.E.D. due to a contaminant layer on the tip apex and differences in energy levels) can be proposed. The second difference between these two average spectra concerns their high-energy cut-offs: a difference of about $0.05 \mathrm{eV}$ is observed. The transmission factor of the potential barrier being very strong for high-energy electrons, a slight difference in I.E.E.D. may be amplified by the transmission factor leading to such offsets in high-energy cut-offs.

Unfortunately, the average spectrum of light emitted by an $\mathrm{AuAu}$ tunneling junction at $2 \mathrm{~V}$ is very difficult to obtain. Indeed, a decrease in emission rate is observed when applying higher than usual bias voltages: light emission can be suppressed on a scanned light-emitting area by applying a bias voltage of typically $2 \mathrm{~V}$ for $\mathrm{Au}-\mathrm{Au}$ tunneling junctions [13]. A possible interpretation of this phenomenon has already been given [14]: this decrease in emission rate can be attributed to the response of the molecules physisorbed on the gold surface to the high electric field applied in the tunneling junction. The bias voltage range available for the study 


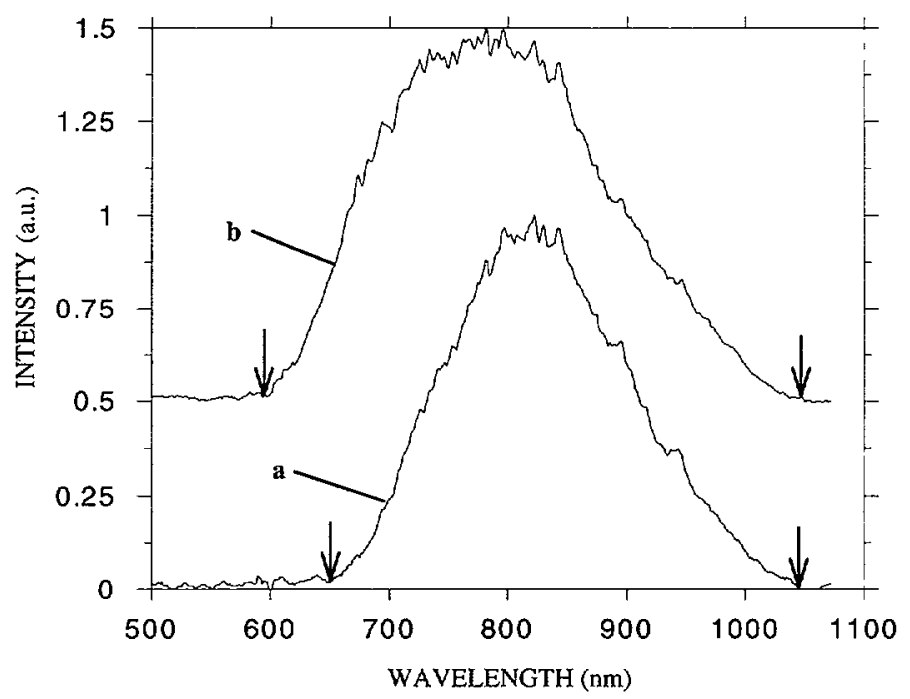

Fig. 7. - Normalized average spectra of light emitted by a PtIr-Au tunneling junction at fixed bias voltages of $1.8 \mathrm{~V}$ (a) and $2 \mathrm{~V}$ (b), tunneling current being set at $10 \mathrm{nA}$. These spectra have been obtained from 45 spectra recorded during scans of different Au surfaces with different PtIr-tips.

of light emission from an Au-Au junction is thus limited by this $2 \mathrm{~V}$-threshold. To increase the available voltage range and obtain a $2 \mathrm{~V}$-average spectrum, the same study has been performed on PtIr-Au junctions. Indeed, the bias voltage threshold leading to a suppression of light emission in the case of PtIr-Au junctions has been found to be greater than the one needed for $\mathrm{Au}-\mathrm{Au}$ tunneling junctions (typically $2.3 \mathrm{~V}$ in this case).

45 spectra of light emitted by $\mathrm{PtIr}-\mathrm{Au}$ junctions have been recorded at a fixed bias voltage of $2 \mathrm{~V}$ and a tunneling current of $10 \mathrm{nA}$. The $1.8 \mathrm{~V}$-normalized average spectrum (previously presented in Fig. 6b) is shown in Figure $7 \mathrm{a}$ with the $2 \mathrm{~V}$-normalized average spectrum obtained using the same data treatment (Fig. $7 \mathrm{~b}$ ). An offset of $0.16 \mathrm{eV}$ can be observed between the two high-energy cut-offs of these average spectra, their sides being roughly the same. When bias voltage is increased, the low-energy tail of the I.E.E.D. is not modified but its high-energy cut-off is blue-shifted. Average spectra given in Figure 7 are thus dependent on applied bias voltage, the distribution of emitted photons being closely linked to the I.E.E.D..

The presence of different components in individual spectra has also been observed by Ito et al. [19] with a different experimental set-up. The authors reported that polycrystalline Au samples scanned by PtIr tips in an U.H.V.-S.T.M. emitted two characteristic wavelengths (620 $\mathrm{nm}$ and $730 \mathrm{~nm}$ ) at a fixed bias voltage of $2.5 \mathrm{~V}$ and a tunneling current of $5 \mathrm{nA}$. Here again, differences in emission wavelengths could partially be due to changes in the I.E.E.D. caused by the oxidization state of the tip apex and/or by the presence of contaminants in the tunneling barrier when the experiment is conducted in air.

\section{Conclusion}

Spectroscopy of light emission from a Scanning Tunneling Microscope in air on Au samples evidences different emission wavelengths in the near-infrared region. Even if the recorded spectra are found to be specific for a given tip-sample configuration, reproducible phenomena 
can be observed. Emission wavelengths are found to be independent of tunneling current in the $5 \mathrm{nA}-100 \mathrm{nA}$ range and thereby of tip-sample distance. The wavelengths of these different spectral components, closely linked to the basic emission mechanism, are found to be independent of bias voltage. However, new spectral components appear and high-energy cut-offs are blue-shifted when the Injected Electron Energy Distribution is modified via applied bias voltage. The results obtained in the case of $\mathrm{Au}-\mathrm{Au}$ and PtIr-Au tunneling junctions show emission spectra to be dependent upon tip materials and applied bias voltage. Injected Electron Energy Distribution seems to play a major role in light emission from a S.T.M..

\section{Acknowledgments}

The authors would like to thank G. Peuch for technical assistance. This work has been supported by the Ultimatech program.

\section{References}

[1] Gimzewski J.K., Reihl B., Coombs J.H. and Schlitter R.R., Photon emission with the scanning tunneling microscope, Z. Phys. B - Cond. Matter 72 (1988) 497.

[2] Berndt R., Photon emission induced by the scanning tunneling microscope, Scanning Microscopy 9 (1995) 687.

[3] Renaud P. and Alvarado S.F., Mapping quantum-well energy profiles of III-V heterostructures by scanning-tunneling-microscope-excited luminescence, Phys. Rev. B 44 (1991) 6340 .

[4] Horn J., Richter R., Hartnagel H.L., Sprössler C.A., Bischoff M. and Pagnia H., Studies of GaAs surfaces by scanning tunneling induced photon emission, Mater. Sci. Engin. B 20 (1993) 183.

[5] Dumas P., Gu M., Syrykh C., Gimzewski J.K., Makarenko I., Halimaoui A. and Salvan F., Direct observation of individual nanometer-sized light emitting structures on porous silicon surfaces, Europhys. Lett. 23 (1993) 197.

[6] Vazquez de Parga A.L. and Alvarado S.F., Magnetic circular dichroism in Cobalt films observed with scanning-tunneling-microscope-excited fluorescence, Phys. Rev. Lett. 72 (1994) 3726.

[7] Lambe J. and McCarthy S.L., Light emission from inelastic electron tunneling, Phys. Rev. Lett. France 37 (1976) 923.

[8] Berndt R., Gimzewski J.K. and Johansson P., Inelastic tunneling excitation of tip-induced plasmon modes on noble-metal surfaces, Phys. Rev. Lett. 67 (1991) 3796.

[9] Persson B.N.J., Baratoff A., Theory of photon emission in electron tunneling to metallic particles, Phys. Rev. Lett. 68 (1992) 3224.

[10] Johansson P., Monreal R., Apell P., Theory for light emission from a scanning tunneling microscope, Phys. Rev. B 42 (1990) 9210.

[11] Berndt R. and Gimzewski J.K., Injection luminescence from CdS(1120) studied with scanning tunneling microscope, Phys. Rev. B 45 (1992) 14095.

[12] Sivel V., Coratger R., Ajustron F. and Beauvillain J., Photon emission stimulated by scanning tunneling microscopy in air, Phys. Rev. B 45 (1992) 8634.

[13] Sivel V., Coratger R., Ajustron F. and Beauvillain J., Control of photon emission by scanning tunneling microscopy in air, Phys. Rev. B 50 (1994) 5628. 
[14] Sivel V., Coratger R., Ajustron F. and Beauvillain J., Interpretation of the control of the photon emission stimulated by STM, Phys. Rev. B 51 (1995) 14598.

[15] Hegner M., Wagner P. and Semenza G., Ultralarge atomically flat template-stripped Au surfaces for scanning probe microscopy, Surf. Sci. 291 (1993) 39.

[16] Landau L. and Lifchitz E., Quantum Mechanics (Mir Editions, Moscow, 1947).

[17] Rendell R.W. and Scalapino D.J., Surface plasmons confined by microstructures on tunnel junctions, Phys. Rev. B 24 (1981) 3276.

[18] Berndt R., Photon emission from the scanning tunneling microscope, PhD Thesis (Basel University, 1992).

[19] Ito K., Ohyama S., Uehara Y. and Ushioda S., STM light emission spectroscopy of surface micro-structures on granular Au films, Surf. Sci. 324 (1995) 282. 